\title{
Aportes metodológicos de las ciencias sociales para la construcciónde políticas públicas en el Quindío Colombia*
}

\author{
Methodological contributions of social sciences for the construction of \\ youth Public policies in Quindío Colombia
}

\author{
Natalia Andrea Pérez Alonso** \\ Luis Felipe Marín GuZMán ${ }^{* * *}$
}

*Esta reflexión es el resultado de la fase inicial del proyecto de investigación "Modelo participativo de formulación de política pública de juventud en el departamento del Quindío" del grupo Paideia de la Universidad La Gran Colombia sede Armenia.

** Magister en Sociedades Rurales. Mg en Antropóloga. Profesora del Centro Ética y Humanidades de la Universidad La Gran Colombia. Correo: perezalonatalia@miugca.edu.co ***Candidato a Maestría en Historia e Historiador. Profesor del Centro Ética y Humanidades de la Universidad La Gran Colombia Correo: maringuzluis@miugca.edu.co

Fecha de recepción: Noviembre de 2019 Fecha de aprobación: marzo de 2020

Para citar este artículo / To reference this article Aportes metodológicos de las ciencias sociales para la construcción de políticas públicas en el Quindío Colombia. Inciso, $22(1)$; 57-74.

DOI: http://dx.doi.org/10.18634/incj.22v.1i.1031

\section{Resumen}

La juventud en el departamento del Quindío, Colombia, es actualmente una de las poblaciones de esta región más vulnerables a las crisis económicas, a las coyunturas globales ambientales y tecnológicas, y a los conflictos regionales y locales; generando cada vez más incertidumbre en los procesos de autoconciencia del lugar sociopolítico que tienen en las sociedades a las que pertenecen. Esta fragilidad se manifiesta en las discontinuidades ejercidas en los mecanismos de participación ciudadana propios de esta población, como lo son las políticas públicas de juventud. A partir de una revisión documental cualitativa, este artículo tiene el objetivo de analizar los diversos aportes metodológicos de las ciencias sociales para el desarrollo y fortalecimiento de los enfoques participativos de construcción de las políticas públicas de juventud del departamento. Esta lectura devela la necesidad de plantear enfoques que garanticen los derechos humanos, la 
justicia social, la inclusión y la apropiación de las capacidades de agencia de los jóvenes para la transformación social. Lo que conlleva a considerar la legitimación de la acción científica como una acción política, y las prácticas socioculturales como prácticas políticas.

Palabras clave: juventud, ciencias sociales, políticas públicas, metodologías, participación.

\section{Abstract}

Youth in the department of Quindío, Colombia, is currently one of the most vulnerable populations in this region to economic crises, global environmental and technological junctures, and regional and local conflicts. Generating more uncertainty in the processes of self-awareness of the socio-political role they have in the society to which they belong. This fragility is manifested in the discontinuities exercised in the citizen participation mechanisms of this population, such as public youth policies. In this way this article aims to analyze the methodological contributions of social sciences to strengthen and develop participatory approaches to the construction of public policies of youth of the department, which guarantees human rights, social justice, inclusion and appropriation of the abilities of the youth to transform the society.

Keywords: youth, social sciences, public policies, methodologies, participation.

\section{Introducción}

En las sociedades contemporáneas existen múltiples disputas culturales y políticas generadas por los desencuentros permanentes entre los diversos modelos económicos, que permean las condiciones sociales sobre las cuales se establecen patrones de comportamiento individual y colectivo que actualmente son cuestionados por las implicaciones directas que estos tienen en el desarrollo de fenómenos como la desigualdad social. En Latinoamérica, estas asimetrías políticas, sociales, económicas y ambientales son objeto un estudio constante de las ciencias sociales, ya que a través de estos procesos se filtran los mayores, si no todos, los problemas que aquejan a nuestras sociedades en todas las escalas, bien sean nacionales, regionales o locales.

Muchos de estos países, desde el reconocimiento de su lugar de enunciación, tienen en cuenta los diversos contextos históricos que han antecedido estas asimetrías, y no solo han generado todo un vademécum de aproximaciones teóricas y conceptuales desde la academia, sino que además se han construido mecanismos sociales y políticos de transformación de estas realidades que ponen sobre la agenda pública la posibles soluciones. En Colombia, el largo y complejo camino que se ha construido como Estado-nación, ha delimitado patrones de participación democrática que conjugan las distintas demandas sociales con las estructuras de gobernabilidad existentes. Uno de estos mecanismos son las políticas públicas, entendidas como: "El conjunto de iniciativas, decisiones y acciones del régimen político frente a situaciones socialmente problemáticas" (Vargas, 2001). Las cuales pueden tener por objeto a una población específica (niños, jóvenes, mujeres, adultos mayores, etc.), o fenómenos sociales como la salud pública, la educación, etc.

Sin embargo, los múltiples tropiezos de la gestión pública nacional, ha potencializado la necesidad cada vez más imperante de integrar nuevos enfoques de colaboración intersectorial que promuevan la construcción participativa de los diversos actores. Ahora bien, en la configuración de una sociedad más 
justa y responsable con la garantía de los derechos humanos, la educación es la base fundamental de ese proceso. Formal o informal, público o privado, cualquier espacio de aprendizaje y socialización es a su vez un espacio de formación de las condiciones de humanidad que pueden posibilitar una existencia humana digna. Por ello, uno de los actores claves en el desarrollo de las políticas públicas son las instituciones de educación superior: las universidades.

En consecuencia, este artículo tiene como objetivo analizar las diversas rutas metodológicas que se pueden desarrollar para fortalecer los enfoques participativos de construcción de las políticas públicas de juventud del departamento, que puedan garantizar los derechos humanos, la justicia social, la inclusión y la apropiación de las capacidades de agencia de los jóvenes para la transformación social.

Este análisis se presenta como una reflexión producida a partir del desarrollo de las actividades de investigación propuestas en la primera fase de ejecución del proyecto "Modelo participativo de formulación de política pública de juventud en el departamento del Quindío" del grupo Paideia de la Universidad La Gran Colombia (sede Armenia), y el Centro de Ética y Humanidades. Este proyecto tiene como objetivo el diseño de un modelo participativo de formulación de política pública de juventud en el departamento del Quindío.

El proyecto metodológicamente se plantea como una investigación social de tipo cualitativo con un enfoque participativo, y precisamente este enfoque es el objeto de estudio al cual nos aproximamos en el desarrollo reflexivo del presente artículo, teniendo en cuenta el marco investigativo en el cual se inscribe. Actualmente la garantía de la "participación" en las investigaciones sociales se ha utilizado para justificar éticamente el compromiso con las poblaciones estudiadas, pero las debilidades en las técnicas o los intereses económicos y políticos de las investigaciones, por lo general, reducen este gran potencial metodológico a la creación de espacios de socialización de los proyectos. Por esta razón, se hace énfasis acá en que la perspectiva participativa del proyecto es comprendida como un enfoque metodológico que asume a los jóvenes como agentes sociales capaces de analizar su propia realidad y de coproducir el conocimiento necesario para la construcción de unos insumos aplicables a la transformación de su propia realidad.

El análisis que se realiza acá, tiene tres ejes: los puntos de encuentro entre los enfoques participativos en las ciencias sociales, las aproximaciones metodológicas de las políticas públicas y la función de la educación para garantizar la participación social y ciudadana. Esto teniendo en cuenta el contexto social, económico y político en el que se desarrolla. El escenario contextual en el cual se inscribe regional y localmente este proyecto es el departamento del Quindío como un territorio que se desarrolla entre la articulación de algunas dinámicas políticas, económicas y sociales como las siguientes:

- Por su ubicación le denominan el triángulo de oro de Colombia, ya que se encuentra geo estratégicamente en un lugar importante del país para la movilidad nacional. (Gobernación del Quindío, 2016). Esto ha generado un desarrollo en infraestructura vial que conlleva posibilidades de empleabilidad para muchos jóvenes.

- Por sus condiciones climatológicas se ubica al departamento como una de las regiones de gran potencial agrícola. Sin embargo, hay un menor número de población menor de 15 años en el sector rural, dando paso al envejecimiento demográfico y generando un bajo porcentaje de relevo generacional del sector. La estructura demográfica se caracteriza en la actualidad por una menor participación de población de niños y jóvenes y una mayor presencia de adultos mayores con respecto a los primeros años del presente siglo. (Gobernación del Quindío, 2016).

- El departamento del Quindío y su capital Armenia son una de las cinco zonas con mayor amenaza sísmica en Colombia. 
- Frente a los grupos de edad más sensibles para los temas de protección y desarrollo, se encuentra que para los próximos años el número de jóvenes entre 20 y 29 años tiende a aumentar, mientras que el volumen de niños, niñas y adolescentes se mantiene estático o disminuye. (Gobernación del Quindío, 2016).

- El Quindío es el departamento del país con mayor tasa de desempleo de acuerdo con los informes del Departamento Administrativo Nacional de Estadísticas - DANE (2018), con una tasa del 13.5\% e impacto directo en la población juvenil constituye uno de los problemas-causas principales de las condiciones y situaciones actuales de la población joven del Quindío.

- Según estudios realizados (García Reinoso, Monsalve Durango, \& Lozano Sandova, 2011) para el departamento del Quindío, se evidencia que en torno a la calidad y cantidad de agua que para el año 2025 el 41\% de la población no contaría con disponibilidad de agua para su consumo.

- El departamento se ha convertido en uno de los 5 principales destinos turísticos del país, lo cual ha determinado un giro radical en las economías agrícolas locales que ha sido sustituidas o complementadas con el sector turismo. Esta dinámica económica ha generado unas pautas de migración importantes entre los jóvenes de zonas rurales a las urbanas. Además de los problemas asociados a esto que afectan igualmente a la población juvenil como prostitución, explotación sexual y microtráfico.

- En cuanto a salud mental, el Quindío es uno de los siete departamentos con las tasas de suicidio más altas. Entre los cuales cabe destacar que también está Risaralda y Caldas. En Colombia el suicidio de niños, niñas, adolescentes y jóvenes (hasta los 24 años de edad) se incrementó en un 35,91\% entre los años 2008 y 2017, según cifras estimadas por el INMLCF.

- De acuerdo al Plan de Desarrollo Departamental del Quindío, la deserción ha aumentado tanto en básica secundaria como en educación superior, la tasa de matrícula ha disminuido en básica primaria, y la calidad de los resultados de las prueba saber 11 ha disminuido. El Quindío presenta un 41,6\% del total de la población graduada en este nivel académico que logran ingresar al mercado laboral en el departamento. Por debajo de Risaralda y Caldas.

Estas, entre otras dinámicas, refuerzan la necesidad de que la academia se corresponsabilice activamente con la articulación entre academia, sector público, privado y sociedad civil en la promoción de investigaciones que no solo informen y analicen los contextos y escenarios sociales que afectan directa o indirectamente a la población juvenil, sino que además planteen estrategias y mecanismos políticos de participación de esta población en la formulación de políticas públicas que garanticen sus derechos.

\section{El carácter participativo de la investigación en las ciencias sociales}

El carácter participativo de la investigación en las ciencias sociales, asumido en gran medida en Latinoamérica es una de las particularidades principales de un desarrollo científico que en los últimos 50 años potencializó, por un lado, las dimensiones holísticas, sistémicas, históricas y humanistas de los enfoques metodológicos, por otro lado, el pensamiento crítico y el compromiso político de la academia con la realidad social. En este sentido, las relaciones de poder entre investigadores e investigados se transforman eliminando la verticalidad de estas, proponiendo praxis y epistemologías que generan relaciones horizontales. Además, se fortalece la construcción de enfoques que reconozcan la relación directa entre conocimiento y poder, donde cualquier acción científica es al mismo tiempo una manifestación del ejercicio del poder con relación a la producción de conocimiento. 


\section{Puntos de inflexión: antecedentes y condiciones históricas}

Este enfoque participativo de las ciencias sociales en Latinoamérica, se puede explicar históricamente como una perspectiva metodológica construida no solo a partir de las transformaciones epistemológicas en las ciencias sociales, sino además a partir de las diferentes movilizaciones sociales, políticas y culturales que emergieron en la década de 1960. De acuerdo con (Rodríguez-Villasante, 2007), las tres fuentes de crítica a las ciencias sociales occidentales tradicionales (positivistas) de la segunda mitad del siglo pasado, son la Investigación Acción Participativa (I.A.P.), las perspectivas marxistas y los diferentes espacios académico-políticos producidos en los seminarios centro europeos provocados por la revolución de mayo del 1968.

Los marxismos fueron una de las principales fuentes de crítica a las ciencias sociales occidentales. De acuerdo con Rodríguez-Villasante (2007) "En las metodologías participativas los "chinos", por ejemplo, hacían hincapié en la "línea de masas", es decir, en aprender de la experiencia de las "masas populares". Es decir, la ecuación del conocimiento es práctica-conciencia-práctica, o materia-conciencia-materia. (...)

En el terreno filosófico esta tendencia se nutre del Engels del Anti-Dühring y del Lenin del materialismo y empiriocriticismo y, sobre todo de la cosificación stalinista de las leyes universales de la dialéctica (incluyendo la de la dialéctica de la naturaleza) de las que la historia sería un campo específico de su aplicación. Una segunda tendencia, que surge en la década de los treinta con la publicación de los Manuscritos de 1844 de Marx y que llega a las interpretaciones "humanistas" que florecen en las décadas de los 50 y 60 y se extiende hasta nuestros días, absolutiza el componente ideológico, humanista, del pensamiento marxiano a expensas de su carácter científico y en cierta medida de su contenido de clase, revolucionario. Una tercera tendencia deja a un lado los problemas ontológicos y antropológicos de las dos tendencias anteriores y se centra en una lectura epistemológica de Marx. El marxismo es definido, ante todo por su "cientificidad" y la "práctica teórica" autosuficiente pasa a ocupar un lugar central. La teoría queda separada de la práctica real, y el aspecto ideológico se desvincula así del aspecto científico. Tal es la tendencia que impulsa Althusser y sus discípulos y que durante la década de los 60 se extiende e influye tanto en los países europeos occidentales como en América Latina." (Rodríguez-Villasante, 2007)

En consecuencia, estos procesos nutrieron los debates académicos y políticos en Latinoamérica producto de las tres grandes expresiones de movilización estudiantil que se gestaron durante la década de 1960 en Norteamérica, Francia y México. La primera de ellas en el campus de la Universidad de Berkeley contra la guerra de Vietnam y la invasión de tropas norteamericanas en Santo Domingo, además de la defensa de las ideas de Martin Luther King como un proceso de reivindicación política de las juventudes de ese momento.

Entre estos hechos de 1964 y los de mayo del 1968 en París, diversas protestas se fueron desarrollando en países como Italia, Inglaterra y Alemania, sin embargo, uno de los más importantes y emblemáticos fue la movilización estudiantil parisina. Esta se llevó a cabo en medio de los debilitamientos del gobierno De Gaulle relacionados con los procesos de independencia de Argelia, además, de las protestas con las que los jóvenes estudiantes parisinos demandaban transformaciones sociales, políticas y económicas que mejorarán las situaciones de desigualdad social y pobreza de Latinoamérica y África.

Paralelamente, en México el movimiento estudiantil también se manifestaba en los campus de la Universidad Nacional Autónoma de México. De acuerdo con (Ibarra Güell \& Bergantiños Franco, 2008):

Éste era un colectivo que, como el de los obreros, campesinos/as, etc., se veía dominado por una política y una economía nacional que venía beneficiando y afianzando a la burguesía burocrática y financiera del país. Al mismo tiempo, además, se encontraban encarcelados trabajadores del ferrocarril, algunos maestros, líderes del movimiento estudiantil de Puebla, Michoacán y Guerrero, campesinos/as acusados/as de 
invasiones de tierras, políticos acusados de terroristas, etc. Este movimiento se nutría, como otros, de la influencia de líderes como Martin Luther King, Ho Chi Min, los protagonistas de la revolución cubana, etc. y de la génesis de unos nuevos patrones, como ya comentamos, musicales, de moda, etc. (Ibíd. p.15)

Estos eventos entendidos hoy como fenómenos sociales de reivindicación de las juventudes, aunque no fueron en consecuencia la solución a las problemáticas sobre las cuales se demandaban cambios sociales, sí funcionó como vehículo de unas inconformidades sociales, económicas y políticas que posibilitaron el cultivo y la emergencia posterior de debates académicos que cuestionaban y criticaban las formas tradicionales de producción científica de conocimiento. Por tanto, estos ejercicios académicos produjeron unas rupturas en los paradigmas clásicos que generaron nuevas propuestas metodológicas que no solo fracturaban las diversas formas de objetivación del conocimiento, sino que además producían nuevas reflexiones en torno a los lugares de enunciación de los investigadores. Esto en el marco de la construcción de las ciencias sociales posibilitó para los procesos latinoamericanos una fuerte articulación entre la ciencia y la política, lo cual a su vez genera una reinterpretación sobre la relación teoría-práctica que en gran medida es afín a las postulaciones sobre la "filosofía de la praxis".

\section{La Investigación Acción Participativa}

La investigación acción participativa (I.A.P.) es una metodología principalmente desarrollada en Latinoamérica a partir de los trabajos desarrollados por el sociólogo colombiano Orlando Fals-Borda (1925-2008) y el antropólogo brasileño Carlos Rodrígues Brandâo (1940-). En Colombia Fals-Borda fue pionero en la investigación sociológica, y fundador con Camilo Torres, de la primera facultad de sociología en 1959. Durante la década de 1960, se llevaron a cabo diversas investigaciones sociales que fueron caracterizando y visibilizando las condiciones de desigualdad económica, las estructuras agrarias, las dimensiones sociales de los conflictos agrarios, las lecturas históricas sobre las revoluciones inconclusas, entre otros. Durante las décadas de 1960 y 1970 se fortalecieron las lecturas críticas a estas realidades de injusticia social.

Antes de que la sociología crítica latinoamericana planteara la I.A.P., ya en 1945 el psicólogo norteamericano de origen alemán Kurt Lewin, había concebido la "investigación acción”.

“Lewin concibió este tipo de investigación como la emprendida por personas, grupos o comunidades que Ilevan a cabo una actividad colectiva en bien de todos, consistente en una práctica reflexiva social en la que interactúan la teoría y la práctica con miras a establecer cambios apropiados en la situación estudiada y en la que no hay distinción entre lo que se investigada, quién investiga y el proceso de investigación (Restrepo Gómez, 2002)".

Posteriormente el antropólogo de Chicago Sol Tax en 1958, fundador de la revista Current Anthropology, fue con sus estudiantes de la Universidad de Chicago uno de los primeros en esbozar con la propuesta de "action-anthropology" (antropología de acción) los inicios de la antropología aplicada, a partir de la cual se reivindica el sentido comprometido de la investigación, pretendiendo que estas no solo se desarrollen por el simple hecho de producir conocimiento.

Durante la década de 1970 se plantea la "sociología comprometida" como una forma de protesta intelectual que Fals-Borda (1970) describe como un proceso que se incubo también con el paso de la sociología filosófico-literaria a la empírica (especialmente en Argentina, Venezuela y Colombia), y el establecimiento de institutos de investigación como la Facultad Latinoamericana de Ciencias Sociales (Flacso), en Santiago de Chile, y el Centro Latinoamericano de Pesquisas em Ciências Sociais, en Rio de Janeiro. Entendida desde la perspectiva del mismo Fals-Borda, como: 
La IAP propone una cercanía cultural con lo propio que permite superar el léxico académico limitante; busca ganar el equilibrio con formas combinadas de análisis cualitativo y de investigación colectiva e individual y se propone combinar y acumular selectivamente el conocimiento que proviene tanto de la aplicación de la razón instrumental cartesiana como de la racionalidad cotidiana y del corazón y experiencias de las gentes comunes, para colocar ese conocimiento sentipensante al servicio de los intereses de las clases y grupos mayoritarios explotados, especialmente los del campo que están más atrasados. (Fals Borda: 1987:5)

En este sentido, los postulados centrales para su desarrollo, siguiendo a Calderón y López (2011) son a) la relación sujeto-objeto, b) la práctica de la conciencia, c) el redescubrimiento del saber popular, d) la acción como elemento central de la formación, y e) la participación:

- La relación sujeto-objeto: La IAP implica una relación no jerarquizada entre el sujeto y el "objeto" de estudio, en donde este último se asume también sujeto activo de la investigación generando una relación más horizontal y no vertical. Esta relación postula una comprensión de la intersubjetividad como un proceso de formación-aprendizaje propia de una investigación con este enfoque.

- La Práctica de la conciencia: "La piedra filosofal de aquella trascendencia de un paradigma a otro radicó en la idea de que el conocimiento para la transformación social no radicaba en la formación liberadora de la conciencia, sino en la práctica de esa conciencia”. (Fals Borda: 1983:2) $)^{1}$

- $\quad$ Redescubrimiento del saber popular: El ejercicio de reconocimiento y validación de los saberes populares, ancestrales y tradicionales de los sujetos deconstruye socialmente los prejuicios acerca de la realidad y genera entre las colectividades estructuras de movilización con propósitos sociales colectivos pensados para intervenir la realidad y transformarla.

- La acción como elemento central de la formación: La praxis política permite la cualificación consciente de la acción del sujeto social (unidimensional y colectivo) y a su vez nutre el trabajo comunitario que posibilita la modificación de las situaciones de pobreza, marginalidad o desigualdad en las que por lo general se encuentran. "Es en la práctica de donde se deriva el conocimiento necesario para transformar la sociedad. Aún más: que así mismo en este paso y de ese sentir de la praxis, también se deriva un saber y un conocimiento científico". (Fals Borda: 1983:2; citado en (Calderón \& López))

- La participación: La participación, desde esta perspectiva es activa y crítica. "Participación es, por lo tanto, el rompimiento de la relación tradicional de dependencia, explotación, opresión o sumisión a todo nivel, individual y colectivamente: de sujeto/objeto a una relación simétrica o de equivalencia”. (Fals Borda: 1987:4)

Esta última, que es en cierta medida, la que más interesa a este trabajo, se convierte entonces en un criterio metodológico coherente con la pretensión política de impactar socialmente desde la investigación con la transformación social. La participación acá dispone de unas relaciones de poder mediadas por el compromiso ético de enfrentar desde la ciencia nuevos dilemas morales producto de los conflictos y crisis generadas por la modernidad, el capitalismo, el desarrollo y la globalización.

\section{Reestructuración de la ciencias sociales y la ciencia posnormal}

Paralelamente a estas miradas críticas que en Latinoamérica se realizaban a la sociología y la antropología, durante la década de 1990, el argentino Silvio Funtowic, (epistemólogo y matemático e investigador

1. Cita tomada de Javier Calderón y Diana López Cardona, “Orlando Fals borda y la Investigación Acción Participativa”, en Pablo Imen, Pablo Frisch y Natalia Stoppani, I Encuentro hacia una Pedagogía emancipadora en nuestra América Latina (Buenos Aires: Centro Cultural de la Cooperación Floreal Gorini, sin fecha y sin paginar). 
formado en Buenos Aires) y el norteamericano Jerome R. Ravetz, (epistemólogo y matemático formado en Swarthmore y en Cambridge), desarrollaban en la Universidad de Leeds en Inglaterra un campo reflexivo de la filosofía de la ciencia: la llamada "ciencia posnormal". Entendida como una estrategia de solución de los problemas en las sociedades de la incertidumbre, como las contemporáneas, es un campo de producción que tiene amplias implicancias "fundamentalmente para el accionar colectivo, responsable ante los problemas planteados por el riesgo ambiental global y la equidad entre pueblos, especies y generaciones" (Funtowicz, 1994). De acuerdo con Cecilia Hidalgo, en el prólogo a la edición Argentina de (Funtowic \& Ravetz, 2000):

La complejidad de tales problemas involucra de manera ahora explícita a muchos agentes hasta hace poco no considerados. Todos los que ponen algo en juego en las decisiones públicas tienen su lugar en el diálogo que tenderá a hallar respuestas y soluciones y su participación adquiere el carácter de esencial. Los expertos científicos o los administradores gubernamentales ya no son os únicos participantes legítimos. Tal situación es de singular relevancia pues la predica de las ciencias sociales, de la ética y de los activistas civiles hace tiempo enfatizaban el lugar de autonomía y de conocimiento de agentes en algún sentido "legos".

La ciencia y el desarrollo técnico-científico siempre han correspondido a los problemas de su propio contexto. Sin embargo, la nueva perspectiva de "sociedad del riesgo" acuñada por Ulrich Beck (1986), reconoce que los cambios de la sociedad industrial y las tensiones generadas por la incapacidad de la modernidad para resolver sus propios problemas, están cargados de un ingrediente especialmente complejo: la incertidumbre. El impacto global de los conflictos ambientales y sociales provocado por el optimismo tecnológico es cuestionado por la fragilidad que tiene la toma de decisiones bajo escenarios de ignorancia e irreversibilidad. Por ello,

“En un estilo dialéctico el sistema tecnológico moderno ha resuelto algunas graves contradicciones en las condiciones materiales y morales de una porción oportunidad de la humanidad, pero al hacerlo ha creado otras contradicciones e incluso más severas y apremiantes como hemos visto tanto en la esfera social como ambientar". (Funtowic \& Ravetz, 2000).

Los dilemas éticos actuales, los conflictos sociales, el desequilibrio ecológico, la desigualdad social, la injusticia ambiental, la pobreza, entre otros, son problemas con condiciones particulares económicas, políticas y culturales que ya no solo se perciben desde perspectivas locales o regionales, sino globales. Por ello, cada vez más emerge la necesidad de sincronizar la ciencia con la política, la economía y la ética, donde se reafirme la complementariedad intrínseca entre el conocimiento científico y los conocimientos alternativos, los saberes tradicionales y lógicas de pensamiento no occidentales. Siguiendo a Jiménez-Buedo y Ramos:

La ciencia posnormal implica investigación en la intersección entre ciencia y política donde las incertidumbres y la carga valorativa son cruciales. Puede ser interpretado como el proceso de elaboración de una política pública en el que se incorporan prioridades, personas, procedimientos, productos y análisis postnormal, extendiéndose también a las fases de implementación y seguimiento. Consecuentemente, dependiendo del contexto en particular, la tarea tendrá más componentes de investigación o de creatividad innovadora de carácter técnico-social. Todo el proceso es en sí un sistema complejo donde se interrelacionan elementos naturales con otros técnicos y sociales. Efectivamente, en nuestra sociedad moderna los científicos y otros profesionales asumen determinados liderazgos en aspectos técnicos que sirven de apoyo a la toma de decisiones políticas. Sin embargo, se viene comprobando en las últimas décadas que únicamente su pericia no resulta suficiente para resolver grandes dilemas que afectan a la ciencia en el mundo actual. Cuando los riesgos no son cuantificables o el daño potencial puede ser irreversible, es imposible hacer recaer en las metodologías hasta ahora utilizadas y las formas tradicionales de conocimiento la orientación de las políticas públicas. Ése es el espacio de la ciencia posnormal, donde la práctica científica puede afrontar los desafíos implícitos en los contextos complejos en los que colisionan pluralidad de actores y valores. Para 
ello se articula -y ésta es la característica más destacable de la ciencia posnormal- la participación pública. (Jiménez-Buedo \& Ramos Vielba, 2009)

A pesar de que la ciencia posnormal se ha concentrado mayoritariamente en los problemas socioambientales globales, además analizados también por la economía ecológica y la ecología política, es crucial reconocer sus aportes como un enfoque en donde se establecen las rupturas epistemológicas necesarias para el contexto global actual. La solución de los problemas actuales no solo debe estar en manos de los científicos y los políticos, es en este sentido, que este enfoque propone "la comunidad de pares ampliada" como la apertura a la participación pública para la resolución de problemas. La también denominada comunidad extendida de pares, rescata no solo implica que el desarrollo científico se reestructure desde la participación democrática, sino que además se potencialice a través del diálogo y la participación de los principales afectados de los problemas contextuales. Lo que Joan Martínez Alier (2000) describe como "ciencia con la gente".

Al parecer, la finalización del siglo XX provocó la necesidad de analizar retrospectivamente el desarrollo científico, las ciencias sociales efectivamente tampoco fueron eximidas de estas miradas que proponían conscientemente una lectura crítica sobre las rupturas y cambios generados por la globalización. En 1995 Immanuel Wallerstein presenta el informe Open the Social Science (Abrir las Ciencias Sociales) en la Social Science Research Council de Nueva York, en el cual se presenta una propuesta de reestructuración de las ciencias sociales. En este informe se plantea que el siglo XIX es la reivindicación de la universidad no solamente en el ámbito de la creación de conocimiento sino también de su reproducción.

En su obra Wallerstein (1996:37) plantea que tres puntos importantes fueron los que definieron a las ciencias sociales después de la Segunda Guerra Mundial: "El primero: el cambio de la estructura política del mundo, el segundo: la expansión de la población y su capacidad productiva y el tercero la expansión geográfica". Así, los nuevos desafíos de las ciencias sociales producen cambios en los objetos de estudio, en las metodologías, en los enfoques epistemológicos, en los impactos de las investigaciones, en el campo de la formación disciplinar y en las dinámicas de institucionalización académica de las mismas.

En el siglo XXI se han asumido los retos postulados en las últimas décadas del siglo XX, sin embargo, las resistencias de los enfoques tradicionales, positivistas generan fuertes choques que se manifiestan como la expresión de las mismas tensiones de la crisis estructural del sistema económico y sus implicaciones ambientales, políticas y culturales.

\section{De las ciencias sociales a las políticas públicas}

Las políticas públicas son hoy uno de los lenguajes predominantes a través de los cuales se expresa la acción del Estado, intentando justamente reconciliar la relación rota y fragmentada que sostiene con el individuo, prestando atención y gestionando las necesidades de los ciudadanos. De hecho muchas de las líneas de acción trazadas por los gobiernos a diferentes niveles se vehiculan a través de estos instrumentos que tienen como condición necesaria dimanar de un ejercicio dialógico permanente entre gobierno - en tanto cabeza del Estado- y la ciudadanía, especialmente la organizada. De acuerdo con Roth (1999) una política pública designa la existencia de

“un conjunto conformado por uno o varios objetivos colectivos considerados necesarios o deseables y por medios y acciones que son tratados, por 10 menos parcialmente, por una institución u organización gubernamental con la finalidad de orientar el comportamiento de actores individuales, o colectivos para modificar una situación percibida como insatisfactoria o problemática". Citado en (Rorth Deubel, 2002). 
Muchos de los estudios, análisis y evaluaciones de política pública terminan enfocándose, prospectiva o retrospectivamente, en los contenidos de su formulación, en los enfoques políticos de los mismos, los alcances de su implementación o en la medición de los impactos logrados. Lo cual es absolutamente necesario, sin embargo, consideramos que esto no necesariamente permite reconocer muchos de los mecanismos de participación ciudadana que los ciudadanos ejercen y desarrollan por fuera de las proyecciones esperadas desde las políticas públicas. Estas dificultades de la participación social y ciudadana han sido objeto de estudio de la ciencia política, la antropología, la sociología, la historia, la piscología social, el trabajo social o la economía. Conllevando al desarrollo no solo de análisis críticos, sino también a la creación de enfoques y metodologías que solucionen estas problemáticas, como ya lo hemos descrito.

Las investigaciones sociológicas y antropológicas sobre las organizaciones sociales, en este caso juveniles, han develado sus procesos históricos de transformación, sus ideologías, sus criterios de organización, sus agendas y demandas políticas y sus identidades culturales; poniendo en evidencia los contextos sociales, políticos y económicos a las que pertenecen. Por ello, consideramos que las coyunturas actuales hacen inevitable la búsqueda de sincretismos entre las metodologías de las ciencias sociales y las de las ciencias políticas.

\section{Enfoques de las políticas públicas}

Teóricamente los enfoques analíticos de las políticas públicas según Roth (2002), se definen a partir de tres grandes posturas teóricas: las teorías centradas en la sociedad, las centradas en el Estado y las mixtas o intermedias. En la primera, el Estado es dependiente de la sociedad y se caracteriza por corrientes tan opuestas como la marxista, neomarxista, las de la teoría de la dependencia, y las racionalistas; en la segunda, se reconoce cierta independencia del Estado, funcionando como un receptor de demandas y un proveedor de servicios y se caracteriza por los enfoques decisionistas y neoweberiano en los que se reduce sustancialmente la influencia de los actores sociales indirectos, así estos sean los afectados por las políticas públicas; en la tercera, se ofrece una postura intermedia con opciones teóricas y políticas diversas como el neocorporativismo, el neoinstitucionalismo, el análisis de redes y la teoría de la acción, entre otros.

En estas dos últimas es importante señalar que se concibe una complejidad social en donde las relaciones de interdependencia entre lo público y lo privado no tienen relaciones de dependencia unidireccional, y sus relaciones son más horizontales que verticales. Entre las corrientes de estos enfoques mixtos se rescatan para lo que acá estamos analizando, la de red o entramado, el neoinstitucionalismo sociológico y el advocacy coalitions. En la primera, se empiezan a reconocer los modelos de interacción que legitiman la ampliación del campo de actores en juego, en donde los actores no institucionales cobran importancia a través de estructuras híbridas de colaboración. En la segunda, de acuerdo con Murell y Serel (1998) se incluye una mirada socio-cultural de las variables de análisis de las organizaciones como criterio de legitimidad de las mismas. En la tercera, el modelo desarrollado por Sabatier (1988) las políticas públicas se entienden como procesos de aprendizaje político conformadas por estructuras de representación social. De acuerdo con Roth (2002) Este enfoque estaría caracterizado por su intento de entender las políticas públicas como matrices cognitivas y normativas que conforman sistemas de interpretación o de representación de la realidad y en los cuales los actores públicos y privados insertan sus acciones.

Siguiendo el ciclo de desarrollo de las políticas públicas que incluyen el surgimiento del problema objeto, el agendamiento, la formulación, la implementación y la evaluación; los anteriores enfoques se contrastan a su vez con los diversos modelos de inscripción del problema en la agenda pública. Estos pueden ser, siguiendo a Roth (2002), por movilización, por oferta política, por mediatización, por 
anticipación, por acción corporativista silenciosa o por la concepción Kingdon. Sin embargo, el proceso de toma de decisión sobre lo que se incluye en la agenda pública requiere posteriormente un proceso de formulación, y además de legitimación a partir del cual se planifica.

Dentro de los enfoques de la planificación, Jurgen Habermas plantea que existen tres tipos ideales de relación entre política, ciencia y ciudadanía: 1) la decisionista, 2) la tecnocrática, y 3) la pragmática. Con respecto a esta última, que es la propuesta del autor, aunque no se desconocen las relaciones entre políticos y científicos, sí se plantean cambios en la lógica de relacionamiento, donde la dependencia unidireccional de uno sobre el otro desaparece, y se plantea primero, la inclusión de un actor: el "público", los ciudadanos; y segundo, el relacionamiento entre estas tres partes se genera por la vía del diálogo interpartes. De acuerdo con (Cano Blandón, 2008) como consecuencia, Habermas (Habermas, 1995) introduce un principio de deliberación societaria, según el cual solo son válidas aquellas normas-acciones que cuentan con el asentimiento de todos los individuos participantes en una discusión racional, el cual pone de relieve la pluralidad de las sociedades contemporáneas. Pluralidad que se pone precisamente en disputa entre los complejos contextos latinoamericanos como los de Colombia.

\section{La participación social y ciudadana}

Los contextos culturales y socioeconómicos son determinantes en términos de cómo se produce la relación ciudadanía-Estado. En el caso del Quindío, ubicado en el Eje Cafetero colombiano, en la zona andina del país, su población se ha caracterizado históricamente por estar ligada a una de las más importantes dinámicas geográficas estratégicas del país generada alrededor de la economía cafetera, donde a su vez confluyen fenómenos como el conflicto armado, el desempleo, los extractivismos y neoextractivismos de la minería, el turismo y la gentrificación. Por ende, comparativamente con otras zonas del país, sus dinámicas presentan enorme fluidez relacional entre lo urbano y rural por lo que plantean un ejercicio de ciudadanía diferente a las regiones exclusivamente urbanas o exclusivamente rurales donde los mecanismos de participación política cambian y están mediados por unos lenguajes y unas pautas de relacionamiento social diferentes.

En el caso de Armenia, la capital del Quindío, los últimos estudios de percepción sobre participación ciudadana, arrojan resultados que plantean la necesidad urgente de estudiar los fenómenos sociales y políticos que provocan estas percepciones y los comportamientos ciudadanos que de allí se desencadenan. Los resultados del tercer estudio de percepción ciudadana en el que se tomó una muestra de 719 personas entre el 1 de noviembre y el 7 de diciembre de 2018, demuestra que hay pesimismo y apatía frente a la institucionalidad pública y la participación ciudadana. Más del $70 \%$ de la población no realiza ninguna acción de participación ciudadana; $43 \%$ de los encuestados percibe que el nivel de corrupción de la ciudad ha incrementado; y solo el 16\% de la población está satisfecha con la gestión de la administración pública; lo cual se traduce en un clima de insatisfacción general de los ciudadanos en su relación con el gobierno.

En virtud de las orgánicas relaciones subsistentes entre la ciudadanía, el contexto y la ética, interesa entonces a este ejercicio plantear un modelo de formulación de política pública de juventud en el departamento, teniendo como línea base la caracterización, reconocimiento y mapeo de actores que se desenvuelven en el campo de las dinámicas juveniles y que asuma como enfoque la participación protagónica de las ciudadanías juveniles. Estas últimas entendidas como la diversidad de prácticas, estéticas, códigos, imaginarios, intereses, formas de organización, legitimación y relacionamiento con los cuales los jóvenes se entroncan e intervienen en la vida social. 
Según Cunill (1999) existen al menos cuatro tipos de participación por las formas de construcción del sujeto social: social, ciudadana, política y comunitaria. De acuerdo con Santillán (2004) Cunill (1999) plantea estos tipos de participación:

La participación social: esta contempla el entorno de la esfera privada y los fenómenos de agrupación a este nivel, en la participación social los sujetos se relacionan individualmente con otras instituciones sociales o, en muchos casos, la organización es mínima.

La participación comunitaria, término que Cunnil vincula con el "desarrollo comunitario" y se refiere a la participación en un cierto nivel que permite alcanzar mejoras inmediatas en las condiciones y niveles de vida; las mismas que se logran por autogestión o iniciativa propia.

La participación ciudadana aquí se muestra un mayor grado de organización e integración, en este nivel los individuos se agrupan y organizan con el fin de intervenir directamente en asuntos de la esfera pública que los afecta.

La participación política, esta participación implica el reconocimiento de la existencia de "intereses públicos" o comunes desde la sociedad civil organizada.

Teniendo en cuenta lo anterior, de hecho, las ciudadanías juveniles se situarían transversalmente en los cuatro tipos de participación, sin embargo, las rupturas que se encuentran en el estado actual de desconfianza y resquebrajamiento de las relaciones entre Estado y sociedad, plantearían la necesidad de postular un modelo de formulación de política pública cuyo enfoque no solo responda al fortalecimiento de la participación política, ciudadana, y a además social. La cual, tendría que ser articulada y constante durante todo el ciclo de política pública, lo que implicaría una amplia y compleja estructura metodológica que garantice pragmáticamente las variables mayoritariamente democráticas entre el cruce de los grados de participación de los ciudadanos y las etapas del ciclo de políticas públicas. Una matriz que puede clarificar y visibilizar fácilmente estos cruces es la diseñada por Cano (2008) como se ve en la tabla 1 :

Tabla 1. Mecanismos de participación ciudadana resultantes de los dos criterios utilizados

\begin{tabular}{|c|c|c|c|c|c|}
\hline \multirow{6}{*}{ 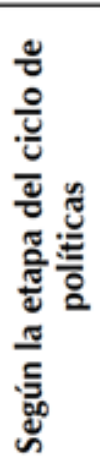 } & & \multicolumn{4}{|c|}{ Según el grado de participación de los ciudadanos } \\
\hline & & $\begin{array}{c}\text { Información } \\
\text { (If) }\end{array}$ & $\begin{array}{c}\text { Consulta } \\
\text { (Cs) }\end{array}$ & $\begin{array}{c}\text { Cooperación } \\
\text { (Co) }\end{array}$ & $\begin{array}{c}\text { Decisión } \\
\text { (De) }\end{array}$ \\
\hline & $\begin{array}{l}\text { Definición del } \\
\text { problema (Dpr) }\end{array}$ & If, Dpr & Cs, Dpr & Co, Dpr & $\mathrm{De}, \mathrm{Dpr}$ \\
\hline & Diseño (Ds) & If, Ds & Cs, Ds & Co, Ds & De, Ds \\
\hline & $\begin{array}{l}\text { Implementación } \\
(I m)\end{array}$ & If, Im & Cs, Im & Co, Im & De, Im \\
\hline & Evaluación (Ev) & If, Ev & Cs, Ev & $\mathrm{Co}, \mathrm{Ev}$ & De, Ev \\
\hline
\end{tabular}

Fuente: (Cano Blandón, 2008) 
Mientras por un lado, se analizan y postulan política y académicamente, nuevas interpretaciones sobre los mecanismos de participación ciudadana, y sus relacionamientos con las otras formas de participación; las ciencias sociales como hemos descrito, también han formulado distintos criterios de determinación sobre las epistemologías metodológicas que pueden hacer que esto sea posible en la práctica y no solo en los discursos que estructuran los sistemas de gobernabilidad.

Sin embargo, estas posturas han emergido muy recientemente durante la segunda mitad del siglo XX, y solo hasta finales del mismo siglo se empezaron a legitimar no solo política sino también científica y socialmente. Por tanto, el desafío más complejo del siglo XXI parece ser su aplicabilidad y esto conlleva procesos de apropiación social que requieren:

1. La formalidad de las estrategias de educación funcionales a estos propósitos de participación ciudadana, social, comunitaria y política.

2. La construcción de ejes de interacción sectorial que faculten a las ciudadanías de un ejercicio participativo activo en todos los procesos del ciclo de las políticas públicas.

3. El diseño y creación de modelos experimentales que respondan a los nuevos conflictos sociales, los dilemas morales, las contingencias ambientales, las crisis económicas y las rupturas caóticas de los sistemas políticos.

4. La reinterpretación de los capitales axiológicos que orientan la valoración de las acciones políticas en la esfera de lo público.

\section{La función de la educación en la garantía de la participación social y ciudadana}

Como se describió, la educación es uno de los principales ejes de construcción de garantías de la participación social y ciudadana. Por ello, las instituciones educativas se convierten en un actor social principal en este proceso y las universidades están llamadas a participar activa y propositivamente en la desarrollo de estos puentes intersectoriales e intergeneracionales. Teniendo en cuenta que el proyecto en el cual se inscribe este análisis, hace parte de las proyecciones de investigación de la Universidad La Gran Colombia, y que la población objetivo de la educación universitaria en su mayoría es juvenil, un tercer interés en el análisis desarrollado es incorporar la perspectiva educativa en la garantía de los mecanismos de participación. Con el propósito de reconocer las condiciones, imaginarios y prácticas que rodean y protagonizan los jóvenes es fundamental una práctica de permanente reflexión y reconocimiento a partir de ejercicios de investigación que además de hacer de la juventud un objeto de estudio, posibilite su participación activa en procesos de análisis y acción que favorezcan el autoreconocimiento participativo de su contexto, sus condiciones, sus situaciones, y las relaciones políticas y socioculturales que entre ellas se tejen.

En el primer apartado, ya se habían mencionado las diversas corrientes y propuestas metodológicas que se han enfocado en la participación de las investigaciones sociales desde la antropología y la sociología. Sin embargo, de acuerdo con (Colmenares E. \& Piñero M., 2008)

“la otra vertiente es más educativa, y está inspirada en las ideas de Paulo Freire (1974) en Brasil, L. Stenhouse (1988) y Jhon Elliott $(1981,1990)$ discípulo de Stenhouse en Inglaterra, así como por Carr y Stephen Kemmis (1988) de la Universidad de Deakin en Australia". En la educación una vertiente caracterizada por la aproximación pedagógica a la participación dialógica es por ejemplo: "el diálogo de saberes". 
Paulo Freire entiende la educación como un encuentro entre seres humanos, mediado por el mundo para pronunciarlo, esto es, para construirlo: "Es un encuentro que solidariza la reflexión y la acción de sus sujetos encauzados hacia el mundo que debe ser transformado y humanizado". (Bastidas , Pérez , Torres, Escobar , \& Arango, 2009)

Si reconocemos que las políticas públicas son herramientas de participación ciudadana que se construyen a través de una red polisignificada y multisituada de actores sociales, políticos, y científicos, es porque concebimos a su vez que los ejes de construcción de las mismas funcionan bajo procesos de apropiación social y aprendizaje que se manifiestan en las relaciones entre lo colectivo y lo individual, lo público y lo privado. Por tanto, un enfoque participativo de esta naturaleza que reconozca la horizontalidad de las relaciones, consideramos que metodológicamente se afianza fácilmente en la propuesta del "dialogo de saberes". Siguiendo a (Pérez Luna \& Alfonzo Moya, 2008):

\footnotetext{
"El diálogo de saberes debe constituirse en el cruce de cultura pública y cultura académica; en este sentido, se debe superar la creencia de que todo conocimiento escolar representa lo válido y lo que proviene de lo cotidiano debe ser rechazado. El diálogo de saberes representa la recuperación del enlace con la realidad, es actividad para que aflore la intersubjetividad como expresión de intercambio entre el tiempo cultural y la necesidad de resignificar el valor del formarse desde una perspectiva de creatividad".
}

Ahora bien, si por "escolar", entendemos lo académico entonces rescataríamos de esta interpretación la necesidad de configurar la validez del conocimiento desde la interacción entre los diferentes tipos de saberes. Lo cual ya se ha planteado epistemológicamente desde la I.A.P., solo que sobre áreas de conocimiento distintas.

Al acercar estas posiciones a los procesos de construcción de política pública de juventud en contextos como los latinoamericanos, cabe anotar, que los mecanismos de visibilización sobre la realidad de los jóvenes latinoamericanos en el escenario público, configuran una lectura compleja y doble sobre sus prácticas. Por un lado, un amplio ejercicio participativo pasivo que difumina una diversidad de acciones desarticuladas aparentemente sin mayor impacto, por otro lado, un ejercicio activo de movilizaciones sociales juveniles con altas posibilidades de impacto pero efímeras y desprestigiadas.

Teniendo en cuenta lo anterior, se propone que la academia institucionalice una investigación centrada en la articulación con el Estado y la sociedad para garantizar herramientas que sirvan: primero, al análisis de implementación e impacto de las políticas públicas de juventud entre su población objetivo; segundo, para entender las formas que han adquirido las ciudadanías juveniles en términos de sus prácticas e imaginarios; tercero, para ubicar los ejes centrales sobre los que debe intervenir para propiciar procesos de acercamiento, recomposición de confianzas y trabajo cooperativo entre ciudadanía organizada e instituciones; cuarto, contribuir a disipar la ya naturalizada desconfianza en lo público condición de posibilidad fundamental para la transformación y revitalización de la condición ciudadana; y quinto, de fundamento para un modelo de construcción, aplicación y gestión participativa de políticas públicas para la juventud.

Si tomamos como muestra representativa, la población de la capital del Quindío, se exponen acá también los principales problemas que los jóvenes identificaron para la formulación de la Política pública de juventud de Armenia (2011-2021). De acuerdo con la caracterización realizada sobre Armenia, los cinco principales problemas identificados son:

- Primer problema: "Altos índices de consumo de sustancias psicoactivas"

- Segundo problema: "Pocas oportunidades brindadas a los jóvenes para: la ocupación del tiempo 
libre, el ingreso a la educación superior, programa de esparcimiento para prevenir el consumo de sustancias"

- Tercer problema: "Riesgo psicosocial en la disfunción familiar"

- Cuarto problema: "Apoyo incipiente de la administración municipal"

- Quinto problema “Desempleo, prostitución"

De estos problemas identificados, el cuarto es el único en el que no se reconoció ninguna acción concreta desarrollada por alguna institución, colectivo, u organización para su solución. De acuerdo con la Política Pública de Juventud de Armenia 2011-2021, el análisis de percepción refleja lo siguiente:

El 56\% de los líderes consideran que el problema que más afecta a los integrantes de sus grupos juveniles, es el insuficiente apoyo económico que reciben para el desarrollo de sus proyectos; el 25\% manifiestan la ausencia de oportunidades en los jóvenes para: la ubicación laboral, cubrimiento del plan obligatorio de salud para el tratamiento y prevención del consumo de sustancias psicoactivas, capacitación para el empoderamiento de los líderes juveniles, la organización de sus grupos; el 15\% manifiestan el deficiente apoyo institucional en lo concerniente a: la inclusión de los grupos juveniles en los programas de gobierno, poca experiencia profesional de los personas encargadas de liderar procesos con jóvenes, situación que desencadena poca credibilidad hacia las instituciones encargadas de dinamizar procesos juveniles y con el $4 \%$ los grupos, quienes consideran la politiquería como el problema que más afecta a los jóvenes de la ciudad. (p.94)

En este sentido, el distanciamiento percibido entre los jóvenes y el Estado, este último representado por la gobernación, la alcaldía y sus correspondientes instituciones, plantea una alta probabilidad de que la participación de ellos no solo en la formulación, sino también la implementación de las políticas públicas sea baja o nula. Por tal razón, se considera urgente el desarrollo de investigaciones que reconozcan el trasfondo de esta problemática, ya que en gran medida el fortalecimiento de estas organizaciones juveniles, sus acciones y sus impactos, dependen de los puentes de articulación intersectorial que se requieren tanto con el sector público como privado.

La participación ciudadana de los jóvenes en el Quindío es reducida, por ello, consideramos que es urgente la creación de espacios socio-culturales y mecanismos políticos que promuevan la generación de soluciones encaminadas a la visibilización de las organizaciones juveniles y sus acciones; y a la formación y aprendizaje crítico de esta población en áreas de conocimiento que sean afín al ejercicio de su derechos de participación.

\section{Conclusiones}

A todo proceso de formulación de una política pública de juventud, le antecede un proceso de investigación como se ha explicado. Investigación que está ligada con la identificación y caracterización del problema y el proceso de agendamiento público de la política. Por lo general, los aportes de las ciencias sociológicas y antropológicas se han aplicado específicamente a esta fase del ciclo de construcción de las políticas públicas a través de técnicas de recolección de información, bien sean con metodologías cuantitativas o cualitativas. Sin embargo, consideramos que aunque la tarea sea compleja, el enfoque participativo activo y crítico es la clave hacia la garantía de una democracia que responda a las coyunturas actuales. Es decir, si bien la democracia no es exclusiva de las sociedades contemporáneas, este se ha perfilado como un paradigma de estructura sociopolítica que intenta transgredir los "órdenes" sistémicos convencionales y tradicionales de la modernidad, desde una perspectiva de larga duración. 
Por tanto, es inminente la creación de nuevas miradas sobre la "participación" social, ciudadana, comunitaria y política, que impliquen el reconocimiento de los desafíos que tiene la planificación pública de las sociedades en medio de diversas crisis e incertidumbres globales. Lo que lleva a suponer que estas posturas deberían asumir la necesidad de implementación de metodologías experimentales que declaran su confianza en la creatividad y la adaptabilidad. Condiciones de hecho, inherentes a procesos de desarrollo humano como la niñez y la juventud. En este orden de ideas, a continuación se plantean las conclusiones derivadas de este análisis reflexivo, que incentivarán las posteriores recomendaciones para el diseño participativo del modelo de política pública del departamento del Quindío:

1. Un modelo de formulación de política pública que pretenda acercarse a la configuración de un sistema democrático que garanticen los hoy reconocidos derechos humanos, implica el reto de integrar a su ciclo de desarrollo un enfoque participativo coherente con su contexto. Este enfoque para contextos latinoamericanos como el del departamento del Quindío no puede solo restringirse a los mecanismos de participación ciudadana institucionalizados por el Estado, sino que deberán ser ampliados a nuevos y legitimados espacios sociopolíticos que sean producto de diseño social, la deliberación societaria, el diálogo de saberes, la colaboración y cooperación intersectorial.

2. Esta integración del enfoque participativo no podrá solo corresponder con el proceso de investigación ex ante, en el ciclo de las políticas públicas, sino que además deberá reinventarse para restaurar los sincretismos disfuncionales que han aumentado la desconfianza de nuestras sociedades en el Estado. Si bien, actualmente existen diversas perspectivas sobre los mecanismos de participación ciudadana en las etapas de formulación, implementación y evaluación, la ignorancia (planteada desde los postulados de la ciencia posnormal) es el primer paso en falso hacia la transformación de las incertidumbres en riesgos proyectados. Y esto, en un contexto con tanta variabilidad demográfica, fragilidad socioeconómica, ecológica y social como la del Quindío solo dibuja un panorama de vulnerabilidades exponenciales. Por tanto, un diseño del enfoque participativo que sea transversal a todo el ciclo de desarrollo de las políticas públicas deberá ser coherente con un tejido dialógico crítico entre enfoques como la teoría de redes, el advocay coalitions, el enfoque pragmático habermasiano; y la investigación acción participativa, la investigación colaborativa y el diálogo de saberes.

3. La investigación para la formulación de políticas públicas desde un enfoque participativo concentra una alta dosis de reflexividad, y análisis crítico sobre la población objeto en este caso los jóvenes. Sin embargo, el desafío mayor será transitar hacia una des-objetivación de los actores afectados, para integrarlos a las tomas de decisiones no burocratizadas ni limitadas solo a los espacios de "registro de información" a través de encuestas, o de "inscripción en la agenda pública" a través de movilizaciones sociales. En este punto, la participación se convertirá pragmáticamente en activa solo cuando esta sea aprehendida conscientemente. Estos procesos de aprendizaje, son los que podrán potencializar los criterios de autoconciencia del lugar sociopolítico que tienen en las sociedades a las que pertenecen.

4. La hibridación de postulados que buscan jugar a una concertación necesaria y urgente entre las posturas invita a ampliar la mirada hacia las formas de apropiación social, como una salida hacia la ubicación de los problemas precisamente entre los nodos de intersección de la gran red de actores y fenómenos que participan en el desarrollo de una política pública. Esta mirada en el caso de las juventudes podrá entonces ser detallada en: i) los niveles de reconocimiento y legitimación que los jóvenes han construido sobre las políticas públicas de juventud, así estos se hayan producido al margen de la misma; ii) los mecanismos de participación ciudadanía garantizados con la implementación de las políticas públicas; iii) las formas de ciudadanía juvenil que se han tejido entre las organizaciones juveniles, al margen del desarrollo de las políticas públicas; iv) los canales de comunicación Estado-ciudadanía que facilitan o debilitan el ejercicio 
de las ciudadanías juveniles; y v) Los contextos que producen y desde los que emergen las ciudadanías juveniles.

Los desafíos son muchos y los riesgos de la "experimentación metodológica" son tan ilimitados como la creatividad humana. Pero, consideramos que allí cuando la acción científica se reconoce críticamente como un acción política, y las prácticas socioculturales se comprenden y asumen como prácticas políticas, los cuestionamientos sobre la delgada línea entre el campo de lo público y de lo privado, suponen el reforzamiento de criterios menos volátiles y ambiguos de los sistemas éticos y de los códigos morales. Luego, entonces sí podremos visualizar la punta del iceberg de una transformación social alineada con la igualdad y la justicia social.

\section{Referencias}

Alcaldía Municipal de Armenia. (2011). Política pública de juventud: jóvenes construyendo ciudad 2011-2021. Armenia: Alcaldía de Armenia. Recuperado el 20 de marzo de 2019, de Política pública de juventud: jóvenes construyendo ciudad 2011-2021,: https://www.armenia.gov.co/ wp-content/uploads/2015/02/POL\%C3\%8DTICA-P\%C3\%9ABLICA-JUVENTUD.pdf

Bastidas , M., Pérez , F., Torres, J., Escobar , G., \& Arango, A. (Marzo de 2009). El diálogo de saberes como posición humana frente al otro: referente ontológico y pedagógico en la educación para la salud. Investigación y Educación en Enfermería, 104-111.

Calderón, J., \& López, D. (s.f.). Orlando Fals Borda y la investigación acción participativa: aportes en el proceso de formación para la transformación. I Encuentro hacia una pedagogía emancipadora en nuestra América. Centro Cultural de la Cooperación Floreal Gorini. Obtenido de https://www. javeriana.edu.co/blogs/boviedo/files/pedagogc3adas-eman-lc3b3pez-cardona-y-calderc3b3n. pdf

Cano Blandón, L. F. (julio-diciembre de 2008). La participación ciudadana en las políticas públicas de lucha contra la corrupción: respondiendo a la lógica de gobernanza. Estudios Políticos(33), 147-177. Obtenido de http://www.scielo.org.co/pdf/espo/n33/n33a7.pdf

Colmenares E. , A. M., \& Piñero M., M. L. (2008). LA INVESTIGACIÓN ACCIÓN. Una herramienta metodológica heurística para la comprensión y transformación de realidades y prácticas socio-educativas. Laurus, 14(27), 96-114. Obtenido de https://www.redalyc.org/pdf/761/76111892006.pdf

Cunill, N. (1999). La reinvención de los servicios sociales en América Latina: algunas lecciones de la experiencia. Reforma y Democracia.

Fals-Borda, O. (2015). La crisis, el compromiso y la ciencia. En O. Fals-Borda, Una sociología sentipensante para América Latina (págs. 219-252). México, D. F. y Buenos Aires: Siglo XXI Editores y CLACSO.

Fals-Borda, O., \& Rodríguez, B. (1987). Investigación Participativa. Montevideo: La Banda Oriental.

Funtowic, S., \& Ravetz, J. (2000). La Ciencia Posnormal. Ciencia con la gente. Barcelona: Icaria Antrazyt.

Funtowicz, S. (1994). "Epistemología política, ciencia con la gente". Serie de documentos e Informes de Investigación No. 178 (pág. 43). Buenos Aires: FLACSO.

García Reinoso, P. L., Monsalve Durango, E. A., \& Lozano Sandova, G. (2011). ANÁLISIS ESPACIAL Y 
TEMPORAL DEL ÍNDICE DE ESCASEZ DE AGUA EN LA CUENCA DEL Río QUINDíO. Revista de investigaciones de la Universidad del Quindío(22), 70-82. Obtenido de http://blade1.uniquindio. edu.co/uniquindio/revistainvestigaciones/adjuntos/pdf/fdf1_N2206.pdf

Gobernación del Quindío. (2016). Plan de Desarrollo Departamental 2016-2019 “En defensa del bien común". Armenia: Gobernación del Quindío.

Habermas, J. (1995). Between Facts and Norms . Cambridge, MA: MIT Press.

Ibarra Güell, P., \& Bergantiños Franco, N. (2008). Movimientos estudiantiles de mayo del 68 a la actualidad. Sobre las experiencias "utópicas" en un movimiento peculiar. En X. Albizu Landa, J. Fernández González, \& J. B. Zubiri Rey, Movimientos estudiantiles: resistir, imaginar, crear en la Universidad (págs. 11-28). Asamblea de Ciencias Sociales por una Universidad crítica.

Jiménez-Buedo, M., \& Ramos Vielba, I. (2009). ¿Más allá de la ciencia académica?: Modo 2, ciencia poscadémica y ciencia posnormal. ARBOR Ciencia, Pensamiento y Cultura, CLXXXV(738), 721-737.

Ocampo, A. (2011). Ciudadanía juvenil, juventud y Estado Discursos de gobierno sobre sus significados. Revista Latinoamericana de ciencias sociales, niñez y juventud [online], 9(1), 287-303.

Pérez Luna, E., \& Alfonzo Moya, N. (2008). Diálogos de saberes y proyectos de investigación en la escuela. Artículos arbitrados, 455 - 460.

Restrepo Gómez, B. (2002). Una variante pedagógica de la investigación-acción educativa. Revista Iberoamericana De Educación, 1(29), 1-10. Obtenido de https://doi.org/https://doi. org/10.35362/rie2912898

Rodríguez-Villasante, T. (2007). Estilos y epistemología en las metodologías participativas. Política y Sociedad No. 44, 123-148.

Rorth Deubel, A.-N. (2002). Políticas Públicas Formulación, Evaluación e Implementación. Bogotá: Ediciones Aurora.

Salazar, C. (2011). Análisis de políticas públicas. Agendas CEPPIA.

Santillán, M. (2004). Criterios Metodológicos para construir tipologías de sistemas de gestión participativa municipal. Quito: FLACSO.

Vargas, A. (2001). Notas sobre el Estado y las políticas públicas. . Bogotá: Almudena.

Villasante, T. R. (2010). Historias y enfoques de una articulación metodológica participativa. CIMAS Cuadernos, 1-18.

Wallerstein, I. (1996). Abrir las ciencias sociales. México: Siglo XXI. 